\title{
Stephen King's Body Worlds: Language Conventions and Creativity in Depicting the Inner Body
}

\begin{abstract}
By Alexandra Nagornaya*
The Inner Body is one of the most promising fields of research in Contemporary Cognitive Linguistics, as it gives the key to understanding the mechanisms enacted in conceptualizing nonevident or quasi-evident phenomena. It has become common practice to note the cognitive impenetrability of the Inner Body which stems from its unique phenomenological features. The experiencer finds him/ herself in a cognitive cul-de-sac and is completely lost for words when it comes to verbalizing events that occur in the internal milieu of the body. The culture the experiencer belongs to serves as a mediator by offering a certain set of ready-made verbal means. Conventional inner-body vocabulary, however limited in number and poor in content, bridges the gap between the global, nondiscursive somatic experience and the linear character of the language. Though unable to cover all the multitude of sensations associated with the life of the Inner Body, it offers the experiencer certain landmarks directing his or her creativity in verbalizing inner-body experience so that the individual inner-body vocabularies are conceptually compatible and mutually understandable.

The given paper traces the main tendencies in the development of conventional inner-body vocabulary drawing on horror fiction by $S$. King. The paper aims to reveal specific cognitive mechanisms that underlie King's creativity and examines the ways in which the writer employs conventional language means and experiments with them, elaborating on common inner-body metaphors by creating new domains and combining different metaphorical models.
\end{abstract}

\section{Introduction}

The Inner Body is a domain which is extremely inhospitable to language. Its resistance to the Word is accounted for by its unique phenomenological properties. Forever present at the background of consciousness, it forms a specific somatic accompaniment to any human activity, remaining for the most part unnoticed, in much the same way as any permanent visual or auditory stimulus is ignored by our conscious mind. When we attempt to focus on the

*Associate Professor, Department of West-European Languages and Translation Studies, Moscow City Teacher Training University, Russia 
internal milieu of the body we discover that we are almost powerless to grasp this invisible, largely inaudible and impalpable, labile, elusive, and uncontrollable reality. The mental ungraspability of the Inner Body prevents it from shaping into a full-fledged object of cognition, and consequently, an object of nomination. Thus, the Inner Body is both a 'conceptual blindspot' and a verbal tabula rasa in Contemporary English. The existing repertoire of language means is scarce, to say the least. It covers only a small fragment of corporeality and refers mainly to the structural parts of the Inner Body and painful sensations. Even in these essential areas, it is far from being exhaustive, leaving the experiencer practically speechless when it comes to translating the 'inarticulate voice of the body' (Biro, 2010:169) into conventional language symbols. The need for more precise verbal means, forces the experiencer to expand and modify the conventional inner-body vocabulary, as well as to offer their own verbal formulae. This complex cognitive and language activity results in the emergence of a relatively stable set of verbal means which form what can be termed Inner-body, or Interoceptive Idiolect. From the linguistic point of view, it appears interesting to reveal the mechanisms that underlie the formation of Interoceptive Idiolect and to define ways in which it correlates with the conventional interoceptive vocabulary, offered by the culture the experiencer belongs to.

This task is far more challenging than it may seem as 'somatic muteness' is a kind of 'speech disorder' that does not spare even the most eloquent and articulate of people who have made the Word their livelihood. Having gone through an extensive body of literary texts, I have revealed a certain anti-body conspiracy: a vast majority of contemporary writers shy away from Body Talk altogether or are content to stay within the limited conventional vocabulary. My efforts were generously rewarded, though, when I turned to S. King's fiction which appeared to be a real treasury of unusual, highly creative and extremely vivid descriptions conveying the most subtle nuances of inner-body experience. In the paragraph to follow, I look into the possible reasons for King's centration on body-related topics and attempt to provide an explanation for his higher-than-average ability to articulate inner-body experience.

\section{The Body in S. King's Fiction}

Stephen King is a universally recognized master of horror fiction and the number one best-selling author working in this particular genre. Lauded by some critics as 'the Charles Dickens of our time' (Bloom, 2009:1), he is bitterly criticized by others for lack of originality and personal style, which, however, hasn't prevented him from gathering a multimillion audience of 'constant readers' and keeping it thrilled, entertained, and utterly mesmerized for almost four decades. King's main literary achievement consists in creating his own complex, multi-dimensional universe, in which the ordinary exists side by side with the supernatural, and the macabre often verges on the ridiculous. 
One of the central elements of this universe is the human body, which has a voice of its own in the complex symphony of King's narrative. S. King is one of the most 'somatic' of all contemporary writers; he copiously refers to the body and demonstrates a remarkable ability to convey the most subtle nuances of subjective bodily experiences and provide varied, vivid and highly individualized descriptions.

This centration on the body can be accounted for by a number of reasons. First and foremost, it can be explained by the specificity of the genre the author represents. Horror is considered to be the most 'physiological' of all literary and cinematographic genres with the exception of pornography (Badley, 1996:4). Its physiological character consists in the various and multifaceted ways it addresses the human body. On the one hand, horror dissects and dismembers the human body, revealing the most secret, the most unsightly sides of its life, explicating them in the Word (Literature) or the Visual Image (Cinema). In this sense, a horror writer is somewhat an anatomist, a literary Gunther von Hagens, aiming to demystify the body and open it to public contemplation. Horror ignores the generally accepted cultural taboo on contemplating the dead body, minimizing the distance between Life and Death and reconstructing through discourse the complex continuum of bodily states: from the living flesh through the suffering flesh to the dead flesh.

On the other hand, high-quality horror, to which King's fiction undoubtedly belongs, is always 'writing from the body': the author describes the experience of dealing with the ambient space through the prism of bodily states emerging in the course of these interactions. The writer, as L. Bradley argues, poses 'as a mass media shaman', 'he textualizes aural, visual, and kinetic sensations, evokes icons from films and television, and narrates in a voice that readers experience viscerally' (Bradley, 1996:1). Thus horror gets us back to pre-rational, somatic modes of cognition, often addressed by contemporary neurologists and intuitively known to 'somatically attuned' individuals. This peculiar intuitive knowledge permeates King's narratives, in which characters get 'visceral hunches' and tend to trust their 'gut feeling':

\section{It came home to him in a visceral way then, knocking him clean out of his own body (Dreamcatcher). \\ He felt a sick little chill rush through his guts, a telephone call from nowhere (Dreamcatcher).}

For S. King, awareness of one's corporeality is not only an integral part of cognitive processes, but an essential prerequisite for an active and joyous life on the whole, a life that is lived to the fullest and brings the greatest satisfaction. Happiness comes with the feeling of being 'viscerally alive' ('Pet Sematary'). King's characters perceive the world in a way that contemporary philosophy knows as 'enactivation'; they are indeed enacted into the environment, confirming M. Merleau-Ponty's famous claims that to perceive something is to make it present with the help of the body (see Merleau-Ponti, 
2012). The human body in King's fiction lives in resonance with the world and immediately responds to the events occurring in it:

The crowd lurched and swayed deliriously toward him, and Johnny felt an answering lurch in his own guts (The Dead Zone).

This resonance can come to a peak when the border between the inner body and the ambient space dissolves and the human soma merges with the world in a single chorus:

I became acutely aware of all the noises inside me and outside me, like some crazy orchestra tuning up to play. The steady thump of my heart, the bloodbeat in my ears like a drum being played with brushes, the creak of sinews like the strings of a violin that has been tuned radically upward, the steady hiss of the river, the hot hum of a locust digging into tight bark, the monotonous cry of a chickadee, and somewhere, far away, a barking dog (The Body).

Another factor which probably contributed to the peculiar somatic mode of King's fiction is the writer's own rich and varied bodily experience. Having first gone through a period of alcohol and drug addiction and later a victim of a road accident, he gained a varied nociceptive experience, which became an object of his own reflection and shaped his understanding of the bodily cataclysms in general.

It should be noted, however, that a personal experience of pain and disease does not necessarily result in the emergence of a rich and varied somatic idiolect, although it largely contributes to the process. Bodily sensations in general and pain in particular are fundamentally non-discursive phenomena, which have been described by some scholars as a sphere 'inherently inhospitable to language' (Biro, 2010:218), 'shattering language"' (Scarry, 1985:5), successfully defying all our attempts at verbalization. The language of pain does not directly arise from the experience of pain. Likewise, the ability to speak about bodily phenomena does not spring from the experience of having a body. It has been suggested that our success in creating the discourse of the body depends on a wide range of individual physiological and psychological features. Particularly notable among them are age and gender, type of physique, personal 'bodily axiology', and the type of cognitive style. The sum total of these features, together with the actual bodily experience, forms a peculiar predisposition, 'attunement' to the perception, differentiation and verbalization of corporeal phenomena. In a sense, we can speak of individual 'interoceptive abilities' (E. Esrock ${ }^{1}$ ) which manifest themselves in the person's higher-than-average ability to detect 'the messages that come from the body' (Fisher, 1973:11) and articulate his or her somatic experience. I believe it wouldn't be an exaggeration to say that in S. King's case these interoceptive abilities have developed to a degree close to a genius. It is by a fortunate

\footnotetext{
${ }^{1}$ http://cm.rpi.edu/pl/people-590/ellen-esrock
} 
coincidence that this genius has been given to a person who managed to put it to good use making the voice of the body, part of his general narrative symphony.

\section{Representation of the Inner Body in S. King's fiction}

S. King's fiction is a brilliant example of how individual creativity can overcome language deficiency without coming into a clash with language tradition.

In S. King's, fiction the Inner Body is represented as a dynamic, constantly changing structure, in which nothing is permanent and nothing is a given. One of the most telling examples is the metaphor of depletion that the writer resorts to when describing some generalized sensation which is not located within a certain organ - a strategy that is not universally accepted in Contemporary English. Cf.:

“What - I don't - "But suddenly he did, and his entire midsection first seemed to turn hollow and then to entirely disappear (Misery).

Deprived of its original, natural content, the body turns into an empty container, which while looking the same on the outside, acquires new, unusual, unlikely content:

He's had a blinding headache. It was draining away now, leaving him feeling strangely hollow inside. He felt a little as if his legitimate innards had been scooped out and replaced with Reddi Wip (The Dead Zone).

Now fear came, entering softly, sifting through the hollow places of his body and filling them up with dirty smoke (Pet Sematary).

An inner-body sensation can be described as a total transformation of the body, which affects the very substance it is made of both on the inside and on the outside:

\section{He felt as if all his body had turned to glass (The Library Policeman).}

More often, however, a sensation described by the metaphorical model of transformation, is of local character and embraces a limited fragment of the inner-body space, which undergoes textural, structural, temperature and other kinds of changes:

... for a moment all of Jud's bones turned to white ice (Pet Sematary).

He tried to get to his feet on legs that had gone to water ... (Pet Sematary). 


\section{His belly seemed to have turned to lead (Salem's Lot).}

It should be noted that this technique cannot be considered the author's innovation, and was probably prompted by the conventional idiom 'his bowels turned to water'. This link is confirmed by the author himself in 'The Body'. The technique is actively used by other writers as well. Cf:: My knees had turned to water (A.-T.Castro. Gunfight on Farside); Stregg's guts turned to ice and he phoned Gleason as soon as he could (L. Erdrich, Gleason). King's personal contribution to the development if the inner-body vocabulary, consists in the expansion and enrichment of the original metaphor. On the one hand, he expands the repertoire of lexical means within the framework of the commonly used thematic groups 'natural elements' (water, ice, fire) and 'chemical elements' (lead). Cf.:

... the strength slips from her legs. They turn to sand and she sits on the bed, the towel falling off her, her wet bottom damping the sheet beneath her (The New York Times at Special Bargain Rates).

$<$ his knee> locked again, turning to something that felt like pig-iron ... (Dreamcatcher).

On the other hand, he creates fresh, unorthodox images, employing lexical units which belong to principally different semantic groups, one of them being 'food': All of his muscles seemed to have turned to macaroni (The Library Policeman).

Behind the obvious lexical differences lies a pervasive cognitive similarity: the Inner Body is conceptualized as a structure whose effective work depends on the stability of the original texture and density. Once the parts that are supposed to be solid acquire fragility, fluidity, or mealiness, the body fails to serve its natural functions and causes discomfort to its 'owner'. The exact type of substance the original structure converts into, is of little relevance once it suggests the necessary lack of solidity and ability to maintain the original shape.

A striking feature of King's 'body talk' is the principal multiplicity of approaches towards the interpretation of the inner body processes. King successfully works with all the metaphorical models of the Inner Body that are relevant for contemporary Anglophone culture. The analysis of the verbal means employed in each particular context suggests that King is equally comfortable and articulate when depicting the body as a

house: The words were there, but they were all blocked up. Absurd or not, Monette had a sudden image of a clogged toilet (Mute).

Frank Jewett felt a horrible sinking sensation in the pit of his belly. It felt like an elevator out of control (Needful Things).

fortress: cancer is "a horrible new invader" (Thinner).

machine: They intend to remove Howard Cottrell's heart and see if it blew a piston or threw a rod (Autopsy Room 4). 
He got up, hands still crossed on his chest, as if to hold the fragile machinery together (Apt Pupil)

state: the elaborated metaphor of a team when describing metabolic processes in the story Stationary Bike: “... It helps at this point to think of the metabolic processes as a work-crew. Men in chinos and Doc Martens. ... Their job is to grab the stuff you send down the chute and dispose of it. Some they send on to the various production departments. The rest they burn. ..." (Stationary Bike).

companion: traitor hands (Needful Things).

biological organism: You got yourself a great big old tumor inside you someplace, all black and juicy, sort of a rotted human toadstool down there in your guts, sucking you dry? (Thinner).

This free alternation of different mental models of the body is one of the trademarks of S. King's interoceptive idiolect, while other 'somaticallyoriented' writers demonstrate a marked preference for only one model. Illustrative of this is K. Kesey's novel 'One Flew Over the Cuckoo's Nest' in which all inner-body states and sensations are described in mechanical terms suggesting a preference for the 'body is a machine' model. Autopathographies and medical texts on the whole generally rely on the 'body is a fortress' model, which manifests itself in the use of military metaphors (see Sontag 1979). By contrast, S. King can use different models even within the same novel and when describing the same character, without creating the impression of a stylistic and conceptual cacophony. By alternating the mental model of the inner body and embedding into it the description of the actual inner-body state, $\mathrm{S}$. King sooner or later gets the mental feedback from the reader, who finally finds a description that resonates with his or her own perception of corporeality.

Another characteristic feature of S. King's interoceptive idiolect is the 'somatoscopic' way of representing the characters' emotive states. Due to the specificity of the genre the writer works in, the most frequently described emotion is fear.

Describing the nature of this emotion, King himself points out that fear looks for 'the place where you, the viewer or the reader, live at your most primitive level', fear 'is not interested in the civilized furniture of our lives' (Danse Macabre). Fear disrupts cultural and behavioral conventions and activates the most ancient, the most primitive perception and response mechanisms. One of these mechanisms is a complex set of physiological processes, whose crucial role in experiencing emotions is confirmed by modern psychology that describes emotions as a highly complex psychosomatic cluster.

King demonstrates a profound understanding of the complex psychosomatic nature of fear. Unlike most of his literary colleagues, he is never content with the metalanguage of fear ('he was frightened/scared /terrified, etc.'). He always concretizes the protagonist's emotive state by pointing to specific somatic reactions. Cf.: 
There was no question about the fear now. It was coiling lazily in her stomach like cold water (The Dead Zone).

Perhaps we were too rough with him, but our muscles had been turned to cables with fear (The Body).

He felt his fear twisting and turning inside him beneath his poker face. Sometimes it was big and panicky, trampling everything: the elephant. Sometimes it was small and gnawing, ripping with sharp teeth: the rat. It was always with him (The Stand).

It is noteworthy that, more often than not, the name of the emotion itself is omitted as the somatic complex that the author describes is quite specific and is easily recognized by anybody who has ever experienced fear:

He approached the bathroom scales, his heart thudding so heavily that it made his eyes throb and his head ache (Thinner); His heart set up a large weak clamor in his chest, and he realized that the pie was tipping to the right (Thinner); Her heart leaped in her chest and began to bang so hard between her ribs that she saw small specks before her eyes, growing and shrinking in time with its beat (Pet Sematary).

In S. King's works the somatic component appears relevant when describing other emotive states as well, such as anger, joy, depression, despair, etc. Cf.:

She was terrified... but she could also feel anger buzzing and drilling through her (Needful Things).

The thick wine of fury buzzed and coursed in his head (The Dead Zone).

... exhilaration flared up in him like a shot of coal oil in embers (Pet Sematary).

What merits particular attention is how the writer describes the somatic markers of emotions and what devices he resorts to.

In most cases S. King provides a description of sensations located within the heart. A change in the pattern of cardiac sensations is quite an objective somatic marker of many emotions, and the specific creativity of the writer is not where he localizes them, but how he describes them. The conventional vocabulary of cardiac sensations is rather poor and has at its basis common and frequently used verbs with the semantics of movement. These include beat, rise, sink, fall, jump, leap as well as verbs that denote movement accompanied by a loud sound (thump, thud).

S. King actively uses the verbal resources provided by the English language and develops them supplementing the conventional descriptions with new, nontrivial details, which enable him to enliven old, worn metaphors: 
His heart was still beating like a triphammer (Needful Things).

His heart leaped up in his throat like a crazy jack-in-the-box (Pet Sematary).

Your heart's already pounding like a premature burial victim beating his fists on the lid of a coffin (Dreamcatcher).

Warren Richardson had not been so badly scared since he was a kid, and his heart took a great, unsteady leap in his chest (The Dead Zone).

More than that, the writer expands the existing thematic groups adding new elements of similar semantics. Thus, for instance, the metaphor of running acquires a vast and varied verbal representation, enriched by units which are not commonly used to describe inner-body sensations. Cf.: ... his heart jogged in his chest but did not leap ... (Pet Sematary); His heart broke into a sudden disorganized gallop (Thinner); He was still terribly weak, and every now and then his heart skittered in his chest (like a man who has stepped in something greasy, he thought), but it was gone, just the same ... (Thinner); He leaned forward, his heart picking up speed (Needful Things).

This active vocabulary expansion is possibly accounted for by King's tendency to personify the organs of the inner body, including the heart. Once personified, the organs acquire the ability to make complex movements which are typical of a living being. This strategy ensures a high degree of verbal freedom in describing inner-body phenomena. The heart, for instance, can vary the speed and manner of running, purposefully moving within the inner-body space (... her heart tried to cram its way up into her throat. (Needful Things)), make complex acrobatic tricks (Brian's heart rose - did more than rise. It soared and did a backover flip (Needful Things)), exercise control over its own functioning (And his disturbed heart seemed to calm down and rediscover something like its old steady beat ... (Thinner)), behave in a way that is typical of living beings (My heart vaulted nimbly into the back of my mouth and crouched there, trembling (The Body)).

King creates a principally new group within the framework of the general metaphor of movement, interpreting an inner-body sensation as 'the flight of the heart': He only walked on, skin cold, mouth hot and arid, heart flying (Pet Sematary). This semantic experiment appears successful, as in spite of an unusual use of the verb, it manages to convey the intensity and the persistent character of the sensation experienced by the protagonist.

A. King resorts to principally the same verbal strategies when describing nociceptive (or painful) sensations that are not associated with certain emotions and are localized not only in the heart, but in other organs as well. $C f$ :

He hasn't been feeling well; his stomach has been kicking up on him again (Needful Things).

Her stomach did another forward roll but this time it didn't stop; it just went on somersaulting over and over and she became aware that she was getting sick (The Dead Zone). 
The vocabulary of nociceptive sensations, however, is not limited by the descriptions of the above-mentioned type. It is exactly in this part of the innerbody vocabulary that King's creative potential is fully displayed.

The conventional vocabulary of nociceptive sensations is extremely limited and is almost exhaustively represented by the well-known McGill's Questionnaire which includes 35 lexical units (see Melzack, 1996:40). Its deficiency is felt by any individual who attempts to adapt the ready-made list of verbal means to the actual bodily suffering he or she is going through at the moment. In the words of D. Biro, 'doctors are uncomfortable with the form's metaphorical language, and patients want even more of it' (Biro, 2010:13).

The need for this 'more metaphorical language' forces the sufferer to expand and modify the conventional inner-body vocabulary and to offer their own verbal formulae in order to represent their actual somatic experience.

S. King successfully copes with this challenge offering a wide range of techniques and verbal means. One of the most unusual devices, which is typical of King's idiolect, is a comparison between two sensations when one is likened to the other. Cf.:

\section{Johnny's head was swelling blackly, expanding like a bladder (The \\ Dead Zone). \\ ... my head was throbbing like a swollen gum with an abscess in it (The Library Policeman).}

The sensation which forms the basis for the comparison is one which due to its physiological nature is familiar to everybody and is experienced in principally the same way (discomfort caused by a full bladder, toothache or pain in the gums, headache, etc.). This technique enables the writer to 'objectify' the sensation which cannot be measured quantitatively or qualitatively and convey to the reader its subjectively experienced properties. King goes a step further and makes descriptions of this kind even more direct and vivid replacing the simile with a more risky but more effective metaphor: When she finished the last chicken, each of her fingers had a migraine headache (The Stand). The metaphor makes the age-specific experience of arthritis more understandable for those who, with some luck, are not likely to get it first-hand in the next three or four decades.

One of the most common techniques in King's prose is describing an inner-body sensation in terms of invasion. The inner-body space can get filled with foreign bodies of organic or non-organic nature, which cause a certain sensation varying from mild discomfort to excruciating pain. $\mathrm{Cf}$.:

... it felt as if there was a thorn lodged somewhere inside him (Thinner).

After almost twenty hours in the dampness, his legs felt as if someone had studded them with rusty nails (Misery). 
The tines of a large chrome fork seemed to be planted deep in her flesh (Needful Things).

These foreign bodies can reside within the inner-body space for a long period of time in a dormant state causing pain only in certain specified conditions:

So he somehow managed to lean over again, awakening rusty bandsaws in his legs as he did so ... (Misery).

The inner body can be invaded by living creatures. I tend to believe that descriptions of this kind are largely inspired by the common 'butterflies-in-thestomach' idiom, which is used to denote the state of nervousness and anticipation. S. King opens the inner body to creatures much less benevolent, which are usually associated with danger and cause disgust:

... now it felt as if a swarm of bees had been loosened in the lower half of his body (Misery).

He stared at the figure, feeling a coldly fluttering swirl of moths in his gut (Thinner).

Painful inner-body sensation can even be attributed to destructive human activity:

"Okay," I said, and I as said it some guy pole-vaulted in my stomach. He dug his pole all the way into my balls, it felt like, and ended up sitting astride my heart (The Body)

S. King actively employs the conventional nociceptive vocabulary of zoomorphic semantics. He, however, is not just a passive follower of a wellestablished tradition. King plays and experiments with the original metaphorical model, offering vivid images abundant in details. His pain is not just 'gnawing' but 'gnawing like sharp weasel's teeth', gnawing in order to satisfy its hunger (that old pain was gnawing his bones again, as fresh and as hungry as it had been on the day when ...) (Needful Things).

Using his favorite technique of personifying bodily phenomena S. King actively develops the zoological metaphor and provides the pain with certain vocal abilities: his pain 'mutters', 'shouts', 'sings', 'bellows', etc.:

The pain began to mutter up and down his legs again, and it was speaking even more loudly from the area of his pelvis - he had been sitting up for nearly an hour, and the dislocation down there was complaining about it (Misery).

Pain - the worst in days - bellowed through his legs, and he screamed (Misery). 
Similarly, the organs in which the pain is localized acquire a voice of their own. This metonymical transfer results in descriptions like The stump was twanging viciously (Survivor Type); His bladder was screaming at him (Here There Be Tygers).

An elaborated zoological metaphor which explicates two aspects of animal behavior simultaneously (the audial and the kinesthetic) is presented in the story 'Ayana':

We cut around the medical center ..., me walking slightly bent over because of the pain, which no longer snarled but still glowered (Ayana).

In his verbal experiments, King goes even further attempting to combine within one description principally different metaphorical models:

zoological and weapon: A spear of hungry pain bolted up her forearm to the elbow (Needful Things).

botanical and zoological: His head throbbed like a tomato full of hot, expanding blood (The Dead Zone).

anthropological and natural element metaphor: ... it might have been the sickness, which was now rolling through her belly in gripping, peristaltic waves (The Dead Zone).

These bold experiments result in the emergence of paradoxical, catachretic descriptions which are able to convey different aspects of the experienced sensation (the degree of its intensity, lability or stability, its localization in the inner-body space, etc.). This technique resonates with the phenomenological specificity of pain as the object of naming, namely with its elusiveness, multidimensionality and paradoxical character (see Jackson 2003).

\section{Conclusion}

It is hardly possible to provide within a single paper an exhaustive description of all the strategies and techniques S. King applies when describing the realm of the Inner Body. I pursued a much more humble goal: to reveal the main tendencies relevant for the formation of individual inner-body vocabulary and to demonstrate how it correlates with the conventional language means provided by the language and cultural environment. It appears that however original and unorthodox King's descriptions might be they still reflect a certain mode of perception which has been worked out by the culture and which is present in the collective cognitive space of the Anglophone community. The examples provided in the paper suggest that $\mathrm{S}$. King is unique in his attunement to the life of the Inner Body, the flexibility with which he switches from one perceptual mode to another and resourcefulness in the choice of language means, but his 'visceral imagination' (Collins 2011) is somewhat exaggerated. 
The repertoire of the techniques he sustainably resorts to includes:

1) alternation of different mental models of the inner body which exist on the collective mental space of the contemporary Anglophone World;

2) personification of the inner body organs and processes;

3) resuscitation of old, worn metaphors through the explication of different components of the described situation as well as through the use of additional lexical units which are paradigmatically related to the components of the conventional metaphor;

4) introduction of new thematic groups of lexical units which represent traditional metaphorical models;

5) combining traditional metaphorical models, creating paradoxical, oxymoronic descriptions;

6) describing subjective, principally unverifiable inner-body sensations through a comparison with sensations that possess a higher degree of universality and 'physiological definiteness'.

Exact, highly detailed and varied descriptions of inner-body states and sensations evoke a direct somatic response in the reader, giving him or her an opportunity to get into the protagonist's hide. It activates the mechanism of empathy and enables the writer to achieve a principally new level of communication with his 'constant reader'. Reading S. King's prose is not just a certain aesthetic experience of assessing a situation; it is living in the situation, being enacted in it, feeling every single aspect of it - an experience in which the reader is involved both intellectually and somatically.

\section{Bibliography}

Badley, L. (1996). Writing horror and the body. The fiction of Stephen King, Clive Barker, and Anne Rice. USA: Greenwood Press.

Biro, D. (2010). Listening to pain. Finding words, compassion, and relief. New York: W.W. Norton \& Company Ltd.

Bloom, H. (2009). Stephen King. USA: Chelsea House Publishers.

Collins, M. R. (2011). Stephen King is Richard Bachman. New York: Overlook Connection Press.

Fisher, S. (1973). Body Consciousness. London: Calder and Boyars.

Jackson, M. (2003). Pain: The science and culture of why we hurt. Toronto: Vintage.

Melzack, R. \& Wall, P. D. (1996). The Challenge of Pain. NY: Penguin Books.

Merleau-Ponti, M. Phenomenology of Perception. New York: Routledge.

Scarry, E. (1985). The Body in Pain. The Making and Unmaking of the World. New York: Oxford University Press.

Sontag, S, (1979). Illness as Metaphor. New York: Vintage Books. 
